\title{
The Runaway Greenhouse: A History of Water on Venus ${ }^{1}$
}

\author{
ANDREW P. INGERSOLL \\ Division of Geological Sciences, California Institute of Technology, Pasadena
}

(Manuscript received 12 June 1969)

\begin{abstract}
Radiative-convective equilibrium models of planetary atmospheres are discussed for the case when the infrared opacity is due to a vapor in equilibrium with its liquid or solid phase. For a grey gas, or for a gas which absorbs at all infrared wavelengths, equilibrium is impossible when the solar constant exceeds a critical value. Equilibrium therefore requires that the condensed phase evaporates into the atmosphere. Moist adiabatic and pseudoadiabatic atmospheres in which the condensing vapor is a major atmospheric constituent are considered. This situation would apply if the solar constant were supercritical with respect to an abundant substance such as water. It is shown that the condensing gas would be a major constituent at all levels in such an atmosphere. Photodissociation of water in the primordial Venus atmosphere is discussed in this context.
\end{abstract}

\section{Introduction}

One of the most useful models in understanding the heat budget of planetary atmospheres is the planeparallel atmosphere in radiative equilibrium with a convective troposphere (Goody, 1964). Sophisticated models of this type have been used successfully to predict the observed thermal structure of the earth's stratosphere, as well as the earth's mean surface temperature (Manabe and Wetherald, 1967). Generally, one takes the composition of the atmosphere as given, and then computes the equilibrium temperature profile under the action of a constant solar flux. For the earth, the most important gases are water vapor, carbon dioxide and ozone, since these have regions of strong absorption in the visible and infrared (IR) portions of the spectrum. However, in the case of water vapor the amount of absorber is temperature dependent, since water vapor in the atmosphere is controlled by cycles of evaporation and precipitation. Accounting for this effect leads to interesting results, some of which are not well understood. The purpose of this paper is to show that when the amount of an absorbing gas in the model atmosphere is determined by its vapor pressure, singularities may result, which represent not only a failure of the model, but also signal a profound change in the physical system which the model represents.

These singularities may be understood in terms of the familiar greenhouse effect. Sunlight penetrates to the surface of the planet more easily than IR radiation escapes because gases such as water vapor are strong absorbers of IR radiation. Provided the atmosphere remains partially transparent in the visible, the surface temperature varies directly as the IR opacity of the

\footnotetext{
${ }^{1}$ Contribution No. 1549 of the Division of Geological Sciences, California Institute of Technology.
}

atmosphere. Singularities may arise if the possibility of feedback exists, where the opacity depends on the surface temperature. This is precisely the situation when the IR opacity is due to a gas whose concentration in the atmosphere is controlled by equilibrium with solid or liquid phases at the surface. These singularities manifest themselves for values of the solar constant greater than a certain critical value. Equilibrium is possible below the critical point, and the calculations of Manabe and Wetherald indicate that this equilibrium is stable. Above the critical point, equilibrium is impossible.

These arguments indicate that each abundant infrared-active substance has associated with it a critical value of the solar constant. When the solar constant is less than the critical value, the substance exists mainly in solid or liquid form on the surface of the planet, but when the solar constant is greater than the critical value, the substance exists primarily as vapor in the atmosphere. Clearly, it would be most interesting to know the critical solar constants for water vapor and carbon dioxide, in order to understand the origin and evolution of the atmospheres of Venus, Mars and the earth. It is almost equally clear that one cannot compute such quantities accurately, but even with fairly simple models one can perhaps decide which planets are supercritical and which are subcritical; the results are insensitive to details of the model because of the extreme sensitivity of the vapor pressure vs temperature relation for water vapor and carbon dioxide.

Leighton and Murray (1966) have argued that water and carbon dioxide in the atmosphere of Mars are both in equilibrium with permanent frost deposits on the surface. In our nomenclature this means that both substances are subcritical on Mars. Water is also subcritical on the earth. Carbon dioxide on the earth is 
rather special, because most of it has dissolved in the oceans and is now buried in sedimentary rocks (Rubey, 1964). Perhaps, if there were no oceans, carbon dioxide would be the major constituent of the earth's atmosphere. On the other hand, carbon dioxide is clearly supercritical on Venus, since apparently all of it now resides in the atmosphere. However, the most interesting question concerns water on Venus. We would like to know whether this planet is supercritical with respect to water, and whether such a circumstance could account for the absence of water on Venus today. These questions are rather speculative, and will be deferred to the last section of this paper. In the next two sections we show how singularities arise for the grey atmospheric model, and in a model based on laboratory absorption spectra. Our aim is not to develop a realistic model of the early Venus atmosphere, if that were possible, but rather to establish that these effects do exist, and eventually to show how they might be relevant to Venus.

\section{The grey model}

In this section we consider a plane-parallel atmosphere with a constant net flux upward from below. Only the longwave (IR) portion of the radiation field is considered; absorption of sunlight is assumed to take place entirely at the ground. The IR opacity is due to a single gas whose absorption coefficient is independent of frequency, and we shall employ the Eddington approximation to compute the temperature profile under conditions of radiative equilibrium (Goody, 1964, p. 332). Let $K$ be the mass absorption coefficient of the absorbing gas; then the optical depth $\tau$ is defined as

$$
\tau=\int_{z}^{\infty} K \rho_{v} d z
$$

where $z$ is the vertical coordinate and $\rho_{v}$ the density of absorbing gas.

With these definitions and assumptions, the radiative equilibrium solution is (Goody, loc. cit.)

$$
\begin{aligned}
B(\tau) & =\frac{F}{2 \pi}\left(1+\frac{3}{2} \tau\right)=\frac{\sigma}{\pi} T^{4}(\tau), \\
B(0) & =\frac{F}{2 \pi} \\
B^{*}\left(\tau^{*}\right) & =\frac{F}{2 \pi}\left(2+\frac{3}{2} \tau^{*}\right) .
\end{aligned}
$$

Here $B=(\sigma / \pi) T^{4}$ is the integrated Planck function, where $\sigma$ is the Stefan-Boltzmann constant and $T$ is the temperature; $F$ is the value of the upward radiative flux; and $\tau^{*}$ is the optical depth at the ground. The atmospheric temperature is determined as a function of $\tau$ from $B(\tau)$; the temperature of the ground is determined from $B^{*}\left(\tau^{*}\right)$. Note that there is a discontinuity of temperature at the ground, since $B^{*}\left(\tau^{*}\right)>B\left(\tau^{*}\right)$. For a solar constant $S=2.0 \mathrm{cal} \mathrm{cm}^{-2} \mathrm{~min}^{-1}$ with an albedo $A=0.4$, the global average of the absorbed sunlight per unit area of the planet is $(1-A) S / 4=0.3$ cal $\mathrm{cm}^{-2} \mathrm{~min}^{-1}$. This must be equal to $F$ if the planet is in thermal equilibrium. From (2b), the corresponding stratospheric temperature is $206 \mathrm{~K}$, and if the optical depth $\tau^{*}$ is $O(1)$, the corresponding surface temperature is $280-300 \mathrm{~K}$, which is reasonable for the earth.

The radiative equilibrium solution (2) is usually assumed to hold in the stratosphere from the top at $\tau=0$ to the beginning of the convective troposphere at the tropopause. In the stratosphere it is reasonable to assume that the absorbing substance is mixed uniformly with other gases. However, if the absorbing substance behaves like water vapor in the earth's atmosphere, then near the planet's surface, and in the convective troposphere, we must assume that the density $\rho_{v}$ is close to the saturation value $\rho_{s}(T)$. Selfconsistency of the model demands that these two solutions join at the tropopause, with temperature and absorber amount continuous. Thus, the radiative equilibrium solution with absorbing substance mixed uniformly must be valid down to the level at which the density $\rho_{v}$ is close to the saturation value $\rho_{s}(T)$, evaluated at that level.

It is important that both temperature and absorber concentration be continuous at the tropopause. The tropopause itself must be in thermal equilibrium, which ensures continuity of temperature. Continuity of absorber concentration follows from the fact that the absorbing substance can only be removed from the atmosphere by condensation and precipitation. The presence of reservoirs of the substance in solid or liquid form at the surface ensures that the atmosphere near the ground be close to saturation. The dynamics of moist convection ensures that the convective troposphere be close to saturation at all levels. Thus, the condition that the base of the stratosphere be close to saturation is due ultimately to the presence of solid or liquid phases at the surface of the planet.

We now pursue the consequences of these assumptions. Let $P$ be the pressure, $\rho$ the density and $m$ the mean molecular weight of the atmospheric mixture. Let the mass fraction $\rho_{v} / \rho$ of absorbing gas be constant in the stratosphere. Then from the hydrostatic relation $d P / d z=-\rho g$, and from (1), we have

$$
\tau=K P_{v} m_{v} / m g,
$$

where $P_{v}$ is the partial pressure of absorbing gas, $m_{v}$ its molecular weight, and $g$ the acceleration of gravity. At the tropopause we set $P_{v}=\mu P_{s}(T)$, where $\mu$ is the relative humidity at the tropopause, and $P_{s}$ the saturation vapor pressure. Then combining (2a) and (3) we 
obtain an implicit relation for the temperature $T$ at the tropopause in terms of $F$ :

$$
\frac{F}{2 \sigma}=T^{4}\left[1+\frac{P_{8}(T)}{P_{0}}\right]^{-1}, \quad P_{0}=\frac{2 m g}{3 \mu K m_{v}} .
$$

To interpret the quantity $P_{0}$, we notice from (3) that the optical depth is 1 at the level where the partial pressure $P_{v}$ of absorbing gas is $m g / K m_{v}$, which is equal to $3 \mu P_{0} / 2$, from (4). Taking $K=0.1 \mathrm{~cm}^{2} \mathrm{gm}^{-1}$, a value appropriate to the 8-20 $\mu$ window of water vapor at STP (Goody, p. 196), and $\mu=100 \%, m=29, m_{v}=18, g=10^{2}$ $\mathrm{cm} \mathrm{sec}^{-2}$, we obtain $P_{0}=8 \mathrm{~mm} \mathrm{Hg}$. This value is merely illustrative, since the IR spectrum of water vapor is anything but grey. However, $8 \mathrm{~mm}$ is also typical of the partial pressure of water vapor near the surface of the earth, which is consistent with an optical depth $\tau^{*}$ of the order unity as required in grey atmospheric models (Goody, p. 332). One should realize, however, that any value of $P_{0}$ within an order of magnitude of $8 \mathrm{~mm}$ might be relevant in applying grey atmospheric models to the earth.

The interesting point in connection with (4) is that solutions exist only for values of $F$ less than a certain critical value (Fig. 1). For $T$ small, the function on the right is proportional to $T^{4}$; for $T$ large, the function is proportional to $T^{4} / P_{s}(T)$. The former is an increasing function of $T$ and the latter a decreasing function, so the expression on the right in (4) must have a maximum value. This maximum value may be computed for any substance such as water or carbon dioxide, provided an appropriate choice of the constant $P_{0}$ can be made. In Table 1, critical values of $F$ for water are given, for a range of $P_{0}$. For comparison, the amounts of incident sunlight averaged over the surfaces of the earth and Venus are 0.50 and $0.95 \mathrm{cal} \mathrm{cm}^{-2} \mathrm{~min}^{-1}$, respectively. Allowing for reflection, the amount absorbed is about $0.30 \mathrm{cal} \mathrm{cm}^{-2} \mathrm{~min}^{-1}$ in each case.

We note that both $F$ and $T^{4}$ vary by about a factor of 2 as $P_{0}$ changes by a factor of 50 . This is because $P_{s}(T)$ is such a strong function of $T$. In fact, for the data of Table $1, P_{s}(T)$ is almost directly proportional to $P_{0}$, but the corresponding changes in $T$ are small.

Eq. (4) places an absolute upper bound on the flux $F$ for the model we have considered. We now show that the presence of a convective troposphere places even more stringent restrictions on $F$. An atmosphere satisfying (2) and (3) is just capable of delivering the necessary upward flux required at each level for radiative equilibrium. Replacing this solution below a certain level by a convective troposphere will tend to

TABLE 1. Maximum outgoing flux $F$ and corresponding temperature $T$ at the tropopause, for water, as functions of the assumed choice of $P_{0}$.

\begin{tabular}{lcccccc}
\hline$P_{0}(\mathrm{~mm} \mathrm{Hg})$ & 1 & 2 & 5 & 10 & 20 & 50 \\
\hline$F\left(\right.$ cal cm-2 $\left.\mathrm{min}^{-1}\right)$ & 0.46 & 0.51 & 0.60 & 0.67 & 0.77 & 0.94 \\
$T\left({ }^{\circ} \mathrm{K}\right)$ & 239 & 246 & 256 & 264 & 275 & 289
\end{tabular}

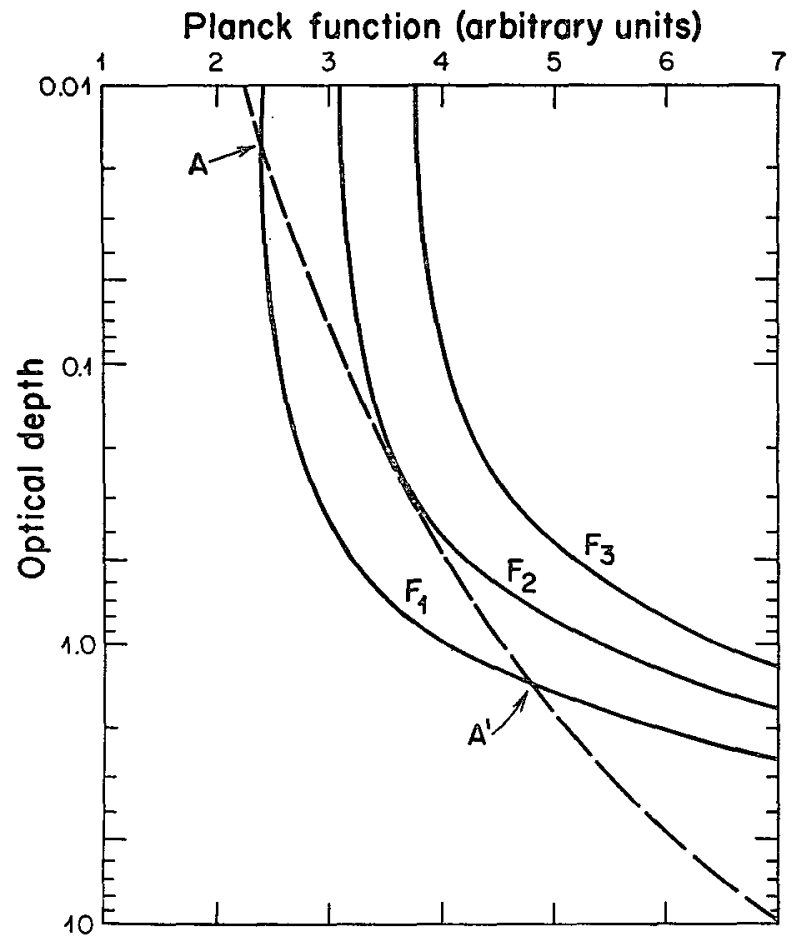

FIG. 1. Planck function $(\sigma / \pi) T^{4}$ vs optical depth $\tau$. The solid curves are for an atmosphere in radiative equilibrium, Eq. (2a) for various $F$. The dashed curve is the saturation relation for a uniformly mixed atmosphere, Eq. (3) with $P_{v}=P_{s}(T)$. For $F=F_{1}$ the curves intersect, and Eq. (4) is satisfied at A. From $A$ to $A^{\prime}$ the radiative equilibrium solution is supersaturated, but this solution must be replaced by a convective troposphere beginning at $\mathrm{A}$. For $F=F_{3}$ the curves do not intersect, and the radiative equilibrium solution cannot be joined to a saturated troposphere. $F=F_{2}$ is the critical case, corresponding to the maximum flux $F$ compatible with Eq. (4).

decrease the upward flux at that level, for several reasons. First, convection will eliminate the temperature discontinuity at the ground, thereby decreasing the surface emission. Second, convection will decrease the lapse rate, so that temperature will increase more slowly with depth. And third, convection will mix more water vapor into the troposphere, thereby increasing the opacity. The last statement only holds when $F$ is close to the maximum value permitted by (4), because only then are the solutions (2) and (3) not supersaturated at some levels (Fig. 1). The net result is that a convective troposphere cannot deliver the necessary upward flux unless the net flux $F$ is some finite amount less than its maximum value from (4). For one specific example (saturated troposphere extending downward to infinity, adiabatic lapse rate, grey absorption independent of pressure), the maximum upward fluxes computed with the Eddington approximation are about $85 \%$ of those given in Table 1 .

\section{Discussion of the runaway greenhouse}

The model we have discussed indicates that equilibrium is impossible when the solar flux exceeds a 
certain critical value, provided the IR opacity is due to a gas whose concentration is controlled by evaporation and precipitation. This concept differs from the greenhouse instability model discussed by Gold (1964), in which a sudden increase in temperature leads to an increase in opacity, which leads to a further increase in temperature, and so on. Such a process is not ruled out by the present calculations, but Manabe and Wetherald (1967) have studied the time evolution of the temperature and water vapor content of the earth's atmosphere, and their solutions tend to approach a stable equilibrium. Unstable equilibrium solutions may exist for some values of the solar constant, but we have shown that equilibrium of any sort is impossible above a certain point.

We now consider what might happen to a planet which is supercritical by the present criterion. In the first place, the albedo of the planet may increase, allowing more sunlight to be reflected, thereby lowering the value of $F$ necessary for thermal equilibrium. This would occur if the cloud cover were to increase, although even for complete cloud cover there would be appreciable absorption of sunlight. For example, the albedo of Venus, which appears to be completely cloud-covered at present, is only about 0.7 , which is roughly the maximum albedo of deep terrestrial clouds. In addition, the conditions under which complete cloud cover is possible are not well known. Terrestrial clouds are water clouds, and the earth is obviously subcritical with respect to water, since the amount of water at the surface is effectively infinite. The composition of clouds on Venus is not known and although water is a possible constituent, it is clear that there are no vast reservoirs of water on the hot surface of Venus. Thus, complete cloud cover might not be possible on Venus if water were as abundant there as it is on the earth.

So let us assume that there is a maximum albedo, significantly less than 1 , determined by the composition of the clouds and by the maximum cloud cover consistent with the atmospheric dynamics. If thermal equilibrium is still impossible according to the model of the last section, then other assumptions must be relaxed. The only alternative is to allow the relative humidity $\mu$ at the base of the stratosphere to fall. In fact, the relative humidity of the earth's lower stratosphere is considerably less than $100 \%$ at mid-latitudes. However, this is because the tropopause is much colder in the tropics than at mid-latitudes, and stratospheric air is apparently dried out as it passes through tropical regions. Thus, in principle, the earth is not an exception to the model, since the water content of the stratosphere is still controlled by the saturation vapor pressure at the tropical tropopause.

If we are to relax the assumption of high relative humidity at the base of the stratosphere, we must invoke an atmospheric model quite unlike the earth's atmosphere. As pointed out in the last section, high relative humidity at the base of the stratosphere is associated with high relative humidity throughout the convective troposphere, and this is ultimately due to the presence of oceans. Thus, if the oceans were to evaporate completely, water vapor would become the major atmospheric constituent, but paradoxically, the relative humidity might fall. The energy needed for evaporating the oceans would only be available if the planet were absorbing more sunlight than it could emit.

This is the runaway greenhouse. For a grey absorbing gas with absorption coefficient independent of pressure, there is no intermediate stage at which thermal equilibrium might be possible. As long as there were oceans, and as long as the troposphere were sufficiently active to mix the vapor upward, thermal equilibrium would be impossible if the solar constant were greater than the critical value. Such a planet would be supercritical with respect to water, and all water on the planet would reside in the atmosphere.

At this stage we might ask whether the effect is due to difficulties with the grey model. The numbers in Table 1 indicate that the greatest values of $F$ are associated with the most transparent cases (large $P_{0}$ ). Thus, in attempting to establish upper bounds on the longwave flux, the transparent portions of the spectrum are the most important. For this reason we choose a value of $K$ for water vapor which is typical of the 8-20 $\mu$ window. It is also clear that if a gas has spectral windows with very low absorption, then the critical values of $F$ will be high. The grey model might not apply as well to carbon dioxide, which shows no measurable absorption between bands, as it does to water vapor, which shows measurable absorption at all IR wavelengths.

It is also possible to compute the maximum upward flux from the troposphere using a radiation chart (Ingersoll, 1968). In this case, however, it is necessary to specify the maximum surface temperature, that is, the temperature at which the greenhouse "runs away." This is essentially the boiling point of the dry atmosphere: as shown in the next section, important changes occur when water vapor becomes a major constituent of the lower atmosphere. For a model of the earth's atmosphere (boiling point $373 \mathrm{~K}$, lapse rate $6.5 \mathrm{~K} \mathrm{~km}^{-1}$, relative humidity $50 \%$ at all levels, carbon dioxide $0.05 \%$ by mass, etc.), the maximum flux from the troposphere is $0.57 \mathrm{cal} \mathrm{cm}^{-2} \mathrm{~min}^{-1}$, computed with an extended version of Yamomoto's (1952) chart. This result is similar to the values of $F$ given in Table 1.

It is clear that in any realistic calculation the critical solar constant will depend on many factors, including the cloud cover and albedo, the composition and structure of the atmosphere, the amount of direct solar heating, and so on. However, there is every reason to believe that a runaway greenhouse will occur under some circumstances, and it is possible that it would occur on Venus if water were abundant there. 


\section{Possible applications to Venus}

We now assume that water was abundant on Venus at one time, and that Venus was supercritical with respect to water. Hoyle (1955) has discussed this possibility, and has argued that the higher water vapor mixing ratios would lead to greater rates of photodissociation. He assumed that hydrogen escaped into space, and that oxidizable material was available at the surface to consume the excess oxygen. Such an explanation accounts for the present low abundance of water and oxygen on Venus without contradicting theories of the formation of the earth's atmosphere (Berkner and Marshall, 1965; Brinkmann, 1969).

Sagan (1960) and Gold (1964) also discussed the possibility of a runaway greenhouse on Venus, but they both concluded that it would not lead to rapid photodissociation compared to that on the earth. A runaway greenhouse would lead to much higher surface temperatures and greater amounts of water vapor in the lower atmosphere, but it would not substantially alter the radiation balance at the stratospheric temperature minimum. Therefore, they reasoned, the amount of water vapor available for photodisociation in the stratosphere would be small, as on the earth, and large amounts of water could not be lost in this way. We shall now show how the total atmospheric pressure in the stratosphere might be substantially reduced in the runaway greenhouse regime, thereby weakening the radiation shield which protects water vapor from photodissociation on the earth.

Here it is necessary to consider the vertical structure of an atmosphere in which water vapor is a major constituent. We assume that the atmosphere is well mixed, and therefore that the mixing ratio is constant in layers where the relative humidity is always low, whether water vapor is a major or a minor constituent. The composition varies only in layers where moist convection occurs, such as the earth's troposphere. We also assume that the vertical structure of such layers resembles the state of a saturated parcel of gas as it rises from the bottom of the layer to the top. In a moist adiabatic process the condensed water remains in the parcel as cloud; in a pseudoadiabatic process the condensed water falls out as rain.

In the Appendix it is shown that the mixing ratio is is nearly constant for both processes when water vapor is a major constituent. If a parcel containing $50 \%$ water is cooled until its temperature is that of the lower stratosphere, the mixing ratio descreases by a factor of 2 or 3 at most. On the other hand, if water vapor is initially a minor constituent, the mixing ratio decreases by many orders of magnitude as the parcel is cooled to stratospheric temperatures (Fig. 2). The latter case is analogous to the earth's atmosphere; the mixing ratio is about $10^{-2}-10^{-3}$ at the surface of the earth, and about $10^{-5}-10^{-6}$ in the stratosphere. The former case is analogous to an atmosphere in which water vapor

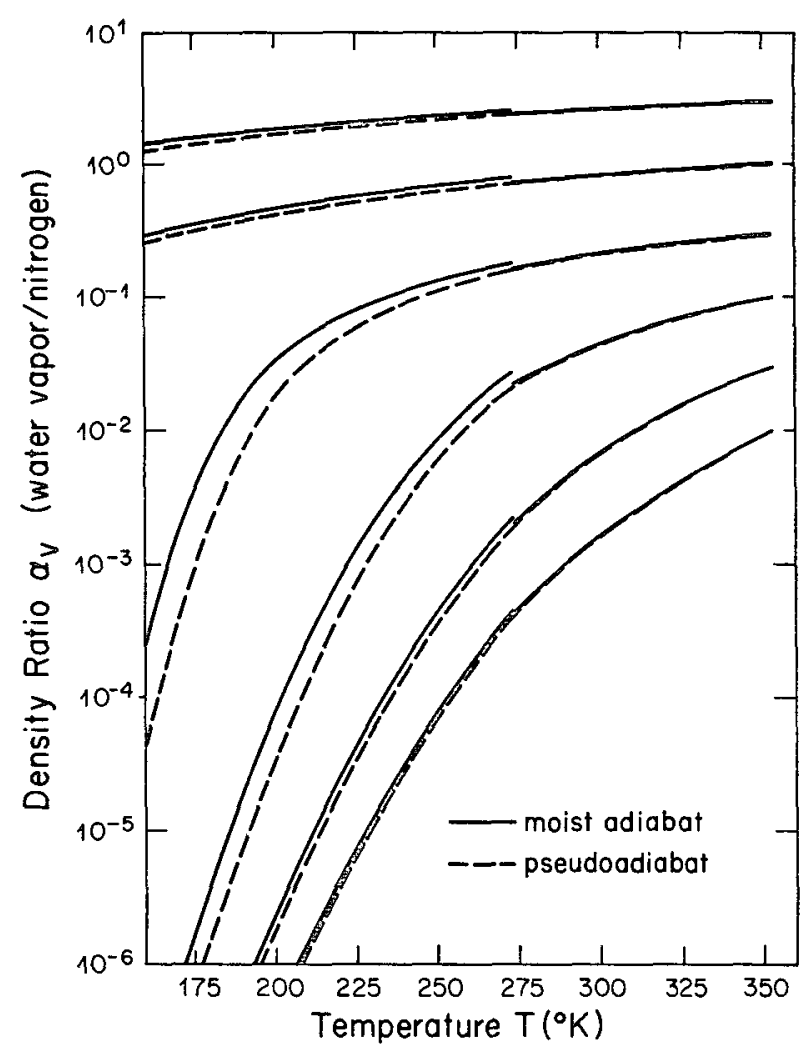

FIG. 2. Water vapor content is temperature for moist adiabatic and pseudoadiabatic expansions. The density ratio $\alpha_{v}$ is the mass of water vapor relative to the mass of noncondensable ideal gas (nitrogen in this case). The initial conditions for the curves shown are $\alpha_{v}=0.01,0.03,0.1,0.3,1.0,3.0$, at $T=353 \mathrm{~K}$, with no liquid water in the system initially.

is a major constituent at all levels. In such an atmosphere the partial pressure of water vapor decreases upward at about the same rate as the total pressure. This means that temperature must decrease very gradually with height. It also means that the total pressure must be very low in the stratosphere, where the partial pressure of water vapor is low. This is an important possibility, which bears on the question of the evolution of the Venus atmosphere.

If water vapor were a major constituent at all levels of the early Venus atmosphere, then it would be one of the principal absorbers of ultraviolet (UV) sunlight. On the earth, most of the UV light is absorbed by noncondensible gases such as molecular oxygen $\mathrm{CO}_{2}$ and $\mathrm{O}_{3}$ ). This absorption takes place high in the atmosphere, where water vapor is extremely rare, so very little energy is available for dissociating water. On Venus the amount of noncondensable gas above the tropopause would be many orders of magnitude less, since the total pressure would be much lower there, so water vapor would be photodissociated at a much greater rate than it is on earth. To put it differently, the cold trap would still operate on Venus, but the radiation shield of other gases above the stratospheric temperature minimum would be absent. 
We may formulate this argument more quantitatively as follows: The cross section of a water vapor molecule is greater than the cross sections of nitrogen, oxygen and carbon dioxide for photons in the wavelength range 1600-2000 $\AA$ (Berkner and Marshall, 1965). Therefore, the fraction of incident photons in this range which are absorbed by water vapor is greater than the water vapor mixing ratio in the upper atmosphere. The photon flux at the earth's orbit in the range $1600-2000 \AA$ is about $10^{13} \mathrm{~cm}^{-2} \mathrm{sec}^{-1}$ (Allen, 1963), which is enough to dissociate about 40 times the amount of water in the earth's oceans during $4.5 \times 10^{9}$ years. On Venus, in the runaway greenhouse regime, a significant fraction of this flux would be available for photodissociating water. On earth only a small fraction is available, since the mixing ratio in the upper atmosphere is $10^{-5}-10^{-6}$.

In this paper we have shown that a runaway greenhouse regime is possible for a planet with oceans at the orbit of Venus. We have also shown that water vapor would be mixed almost uniformly in such an atmosphere, and that large amounts of water would be photodissociated in a relatively short time. It is beyond the scope of the paper to discuss the escape of hydrogen or the formation of oxides. Oxygen may have combined with ferrous iron on Venus to form ferric oxide, or with atmospheric hydrocarbons to form carbon dioxide. High surface temperatures in the runaway greenhouse regime might have hastened this process. There are still many uncertainties in this explanation, but it has the advantage that a major difference between earth and Venus is explained as a necessary consequence of their relative positions in the solar system.

Acknowledgments. The original version of this paper was presented at the 49th Annual Meeting of the American Geophysical Union (Ingersoll, 1968). Since then it has been rewritten several times. Prof. Richard Goody recognized the essential simplicity of the concept described, before it had been described simply, and offered advice and encouragement when it was sorely needed. Financial support was provided by the National Aeronautics and Space Administration under Grant NGL 05-002-003.

\section{APPENDIX}

\section{Condensation of a Major Constituent}

Consider a mixture of water vapor, condensed water (solid or liquid) and noncondensable gas in thermodynamic equilibrium. Let $\alpha_{v}$ be the mass of water vapor relative to the mass of noncondensable gas in the mixture, and let $\alpha_{0}$ be the mass of condensed water relative to the mass of noncondensable gas; $\alpha_{v}$ may take any value initially, but we consider only processes in which the vapor is saturated. During a moist adiabatic expansion the condensed water remains in the system, so the quantity $\alpha_{v}+\alpha_{c}$ is constant, while during a pseudoadiabatic expansion the condensed water leaves the system as soon as it appears, so $\alpha_{c}$ is always zero. We wish to investigate how $\alpha_{v}$ and $\alpha_{c}$ behave as functions of temperature, as the system cools adiabatically or pseudoadiabatically.

The entropy of the system, per unit mass of noncondensable gas, is

$$
s_{n}+\alpha_{v} s_{v}+\alpha_{c} s_{c},
$$

where $s_{n}, s_{v}$ and $s_{c}$ are the specific entropies of noncondensable gas, water vapor and condensed water, respectively. We assume that the specific entropies of the constituents of the mixture are the same as for each substance separately. Thus, since the two phases of water are in equilibrium, the quantities $s_{v}$ and $s_{c}$ are known functions of the temperature $T$. The quantity $s_{n}$ is a function of $T$ and $\rho_{n}$, where $\rho_{n}$ is the density of noncondensable gas. However, the gaseous constituents occupy the same volume, so we have

$$
\rho_{n}=\rho_{v} / \alpha_{v}
$$

where $\rho_{v}$ is the density of saturated water vapor, a known function of temperature. Thus, $s_{n}$ may be considered a known function of the ratio $\alpha_{v}$ and the temperature $T$. Near the critical point of water $(647 \mathrm{~K})$ it may not be valid to treat each constituent separately, so we will restrict our discussion to ranges of temperature considerably below this value.

During an adiabatic expansion the total water and the total entropy are constant; thus

$$
d \alpha_{c} / d T=-d \alpha_{v} / d T
$$

$$
\begin{aligned}
\frac{\partial s_{n}}{\partial T}+\frac{1}{\alpha_{v}} \frac{\partial s_{n}}{\partial \rho_{n}} & {\left[\frac{d \rho_{v}}{d T}-\frac{\rho_{v}}{\alpha_{v}} \frac{d \alpha_{v}}{d T}\right] } \\
& +\alpha_{v} \frac{d s_{v}}{d T}+\alpha_{c} \frac{d s_{c}}{d T}+\left(s_{v}-s_{c}\right) \frac{d \alpha_{v}}{d T}=0 .
\end{aligned}
$$

In addition, if the noncondensable gas behaves as an ideal gas, we have

$$
\begin{gathered}
T \frac{\partial s_{n}}{\partial T}=c_{n} / m_{n}, \\
\rho_{n} \frac{\partial s_{n}}{\partial \rho_{n}}=-R / m_{n},
\end{gathered}
$$

where $c_{n}(T)$ is the molar heat capacity of the ideal gas at constant volume, and $m_{n}$ its molecular weight. Eq. (A4) is then a first-order differential equation for $\alpha_{v}(T)$, i.e.,

$$
\frac{d \alpha_{v}}{d T}=\frac{\left\{\frac{R}{m_{n}}\left[\frac{d\left(\log \rho_{v}\right)}{d T}\right]-\frac{c_{n}}{T m_{n}}-\alpha_{v} \frac{d s_{v}}{d T}-\alpha_{c} \frac{d s_{c}}{d T}\right\}}{\left[s_{v}-s_{v}+R /\left(\alpha_{v} m_{n}\right)\right]},
$$


to be solved subject to the condition

$$
\alpha_{v}(T)+\alpha_{c}(T)=\alpha_{v}{ }^{\prime}+\alpha_{c}{ }^{\prime} \text { (moist adiabat), }
$$

where $\alpha_{v}{ }^{\prime}$ and $\alpha_{c}{ }^{\prime}$ are the initial values of $\alpha_{v}$ and $\alpha_{c}$ at temperature $T^{\prime}$.

In a pseudoadiabatic expansion the condensed water leaves the system, although the entropy of condensation remains. Thus, the appropriate equation is still (A7), but in place of (A8) we have

$$
\alpha_{c}(T)=\alpha_{c}{ }^{\prime}=0 \text { (pseudoadiabat). }
$$

Eqs. (A7) and (A9) describe an irreversible process involving condensation and precipitation, but not evaporation. Therefore, only negative changes in $\alpha_{v}(T)$ have physical significance.

For $T \geq 0 \mathrm{C}$, steam tables (Keenan and Keyes, 1936) were used to obtain values of $s_{v}-s_{c}, d s_{v} / d T, d s_{c} / d T$, and $d\left(\log \rho_{v}\right) / d T$. The quantity $s_{v}-s_{c}$ was taken directly from the tables; the derivatives were evaluated using a linear approximation with a step size $\Delta T=2 F$. Eq. (A7) was divided through by $\alpha_{v}$, and the result was integrated with $\log \alpha_{v}$ as dependent variable. The RungeKutta method was used with a step size $\Delta T=10 \mathrm{C}$ (half step size $5 \mathrm{C}$ ). The equation was integrated with temperature decreasing from the initial temperature $T^{\prime}$ to the point $T=0 \mathrm{C}$.

At $T=0 \mathrm{C}$, all liquid water in the system was assumed to freeze. The total entropy (A1) is conserved during this process, but $s_{c}, \alpha_{c}$ and $\alpha_{v}$ change discontinuously. Therefore, the quantity,

$$
\left(R / m_{n}\right) \log \alpha_{v}+\alpha_{v} s_{v}+\alpha_{c} s_{c},
$$

must be the same before and after freezing, which follows from (A2), (A6), and the fact that $T$ and $\rho_{v}$ are constant. This conservation law is sufficient to determine the change in $\alpha_{v}$ at $0 \mathrm{C}$ when there is liquid water in the system.

For $T<0 \mathrm{C}$, ice was assumed to form directly from the vapor, and the following relations were used:

$$
\begin{aligned}
T\left(s_{v}-s_{c}\right) & =\lambda, \\
T d s_{c} / d T & =c_{i}, \\
T d s_{v} / d T & =-\lambda / T+d \lambda / d T+c_{i}, \\
T d\left(\log \rho_{v}\right) / d T & =\lambda m_{v} /(R T)-1,
\end{aligned}
$$

where $\lambda$ is the latent heat of sublimation, $c_{i}$ the specific heat of ice, and $m_{v}=18$ is the molecular weight of water. Eqs. (A11) and (A12) are the definitions of $\lambda$ and $c_{i}$; Eq. (A13) is the derivative of (A11); and (A14) is the Clausius-Clapeyron equation in which the specific volume of ice has been neglected, and the vapor has been treated as an ideal gas. The values of $\lambda$ and $c_{i}$ were obtained at intervals of $10 \mathrm{C}$ from the Smithsonian Meteorological Tables (1958). The integration of (A7) was continued from $T=0 \mathrm{C}$ to $T=-100 \mathrm{C}$ using these data.
A sample of the results is shown in Fig. 2, for water vapor mixed with nitrogen. The moist adiabats start at $T=353 \mathrm{~K}$ with no liquid water in the system. They are discontinuous at $0 \mathrm{C}$, where the liquid water freezes. The pseudoadiabats have discontinuities of slope at $0 \mathrm{C}$. Curves starting at $T=403$ and $453 \mathrm{~K}$ are similar in every respect to the curves in Fig. 2.

When water vapor is a major constituent $\left(\alpha_{v}>0.1\right.$ in Fig. 2), the function $\alpha_{v}(T)$ is algebraic; $\alpha_{v}$ decreases by a factor of $2-3$ as $T$ decreases from 353 to $173 \mathrm{~K}$. When water vapor is a minor constituent $\left(\alpha_{v}<0.01\right.$ in Fig. 2), the function is exponential with logarithmic decrement about $10 \mathrm{~K}$; in this case $\alpha_{v}$ decreases by several orders of magnitude as $T$ decreases from 353 to $173 \mathrm{~K}$. An asymptotic analysis of (A7), using the approximate formulas (A11)-(A14) with $\lambda m_{v} /(R T) \gg 1$, substantiates these conclusions. Thus, the water vapor content of a mixture will either remain high or decrease exponentially during a moist adiabatic or pseudoadiabatic expansion, depending on whether the vapor is a major or minor constituent.

An interesting peripheral issue concerns the sign of $d \alpha_{v} / d T$ in (A7). Brunt (1934) and McDonald (1964) have speculated that clouds might form on downdrafts under some circumstances. To investigate this possibility we set $\alpha_{c}=0$, corresponding to the initial stage of cloud formation, and we look for negative values of $d \alpha_{v} / d T$, corresponding to condensation ( $\alpha_{v}$ decreasing) during adiabatic compression ( $T$ increasing). Data for saturated water vapor are given by Keenan and Keyes (1936); data for saturated ammonia, carbon dioxide, methane, acetylene, ethane and propane are given by Din (1962).

For all of these vapors, $d s_{v} / d T$ is negative, so the pure vapor $\left(\alpha_{v}\right.$ large) condenses only on updrafts ( $d \alpha_{v} / d T$ positive). A necessary condition for condensation on downdrafts is

$$
T d\left(\log \rho_{v}\right) / d T<c_{n} / R,
$$

which follows from (A7) with $\alpha_{c}=0 ; c_{n}$ is the molar heat capacity of the noncondensable gas at constant volume. For an ideal gas with six internal degrees of freedom, $c_{n} / R$ is 4.5 ; for most real gases at moderate temperatures, $c_{n} / R<4.5$. However, the values of $T d\left(\log \rho_{v}\right) / d T$ for saturated vapors of real substances are never $<7$ (Table 2), so we conclude that real clouds

TABLe 2. Minimum values of $W=T d\left(\log \rho_{v}\right) / d T$ for saturated vapors of various substances. $T_{\min }$ and $P_{\min }$ are the values of the temperature and vapor pressure at which $W$ is a minimum.

\begin{tabular}{cccc}
\hline Substance & $\begin{array}{c}T_{\min } \\
\left({ }^{\circ} \mathbf{K}\right)\end{array}$ & $\begin{array}{c}P_{\min } \\
(\mathrm{atm})\end{array}$ & $W_{\min }$ \\
\hline $\mathrm{H}_{2} \mathrm{O}$ & 530 & 39 & 9.1 \\
$\mathrm{NH}_{3}$ & 330 & 24 & 8.8 \\
$\mathrm{CO}_{2}$ & 250 & 19 & 8.2 \\
$\mathrm{C}_{2} \mathrm{H}_{2}$ & 240 & 10 & 8.1 \\
$\mathrm{C}_{3} \mathrm{H}_{8}$ & 295 & 8.6 & 7.9 \\
$\mathrm{CH}_{4}$ & 125 & 2.7 & 7.7 \\
$\mathrm{C}_{2} \mathrm{H}_{6}$ & 250 & 13 & 7.5 \\
\hline
\end{tabular}


will form only on updrafts. McDonald's criterion may be derived from (A15) using the approximate form of the Clapeyron equation (A14), but this equation is valid only at low temperatures and pressures. McDonald has applied this approximation to saturated vapors close to their critical points, where (A14) is certainly invalid.

\section{REFERENCES}

Allen, C. W., 1963 : A strophysical Quantities, 2 ed. London, Athlone Press, p. 127.

Berkner, L. V., and L. C. Marshall, 1965: On the origin and rise of oxygen concentration in the earth's atmosphere. J. Atmos. Sci., 22, 225-261.

Brinkmann, R. T., 1969: The dissociation of water vapor and evolution of oxygen in the earth's atmosphere. J. Geophys. Res., 74 (in press).

Brunt, D., 1934: The possibility of condensation by descent of air. Quart. J. Roy. Meteor. Soc., 60, 279-284.

Din, F., 1962: Thermodynamic Functions of Gases, Vols. 1-3. London, Butterworths.

Gold, T., 1964: Outgassing processes on the moon and Venus. The Origin and Evolution of Atmospheres and Oceans, Edited by Brancazio and Cameron, New York, Wiley, 249-256.
Goody, R. M., 1964: Atmospheric Radiation. I. Theroetical Basis. London, Oxford University Press, $436 \mathrm{pp}$.

Hoyle, F., 1955: Frontiers in Astronomy. London, William Heinemann, 68-72.

Ingersoll, A. P., 1968: The runaway greenhouse: A history of water on Venus. Trans. Amer. Geophys. Union, 49, 250 (abstract).

Keeman, J. H., and F. G. Keyes, 1936: Thermodynamic Properties of Stream. New York, Wiley, $89 \mathrm{pp}$.

Leighton, R. B., and B. C. Murray, 1966: The behavior of carbon dioxide and other volatiles on Mars. Science, 153, 136-144.

Manabe, S., and R. T. Wetherald, 1967: Thermal equilibrium of the atmosphere with a given distribution of relative humidity. J. Atmos. Sci., 24, 241-259.

McDonald, J. E., 1964: On a criterion governing the mode of cloud formation in planetary atmospheres. J. Atmos. Sci., 21, 76-82.

Rubey, W. W., 1964: Geologic history of sea water. The Origin and Evolution of Atmospheres and Oceans, Edited by Brancazio and Cameron, New York, Wiley, 1-63.

Sagan, C., 1960: The Radiation Balance of Venus. California Institute of Technology, Jet Propulsion Lab., Tech. Rept. No. 32-34, 23 pp.

Smithsonian Institution, 1958: Smithsonian Meteorological Tables, 6 Rev. Ed. Washington, D. C., Smithsonian Institution, p. 343.

Yamomoto, G., 1952: On a radiation chart. Sci. Rept. Tohoku Univ., Series 5, Geophys., 4, 9-23. 